\title{
Expression of Proliferating Cell Nuclear Antigen (PCNA) in Breast Carcinoma and its Association with Ki67 and Tumor Grade in Mastectomy Specimens
}

\author{
Kalaivani Amitkumar ${ }^{1 *}$, Bhuvanamhadevi R ${ }^{1}$, Anandhalakshmi Swaminathan ${ }^{2}$, Saranya Babu $^{1}$ and Balaji $\mathbf{R}^{3}$ \\ ${ }^{1}$ Department of Pathology, SRMMCH \& RC, Kattankulathur, India \\ ${ }^{2}$ Department of physiology, SRMMCH\&RC, Kattankulathur, India \\ ${ }^{3}$ Department of Social and Preventive Medicine, SRMMCH\&RC, Kattankulathur, India
}

\begin{abstract}
Background: Breast cancer ranks first among cancer in urban population of India, and 2nd most common in the rural population. Proliferative activity acts as an adjunct and gives prognostic importance in breast cancer. However, the best method for measurement of proliferative rate is controversial. The aim was to evaluate the association between PCNA labeling index (PCNA-LI) and Ki67 labeling index (Ki67-LI) and their correlation with histological grade.

Methods: Seventy cases of simple and Modified Radical Mastectomy specimens with the diagnosis of carcinoma breast were included in the study. H\&E stained sections were examined for assessment of histological grade. IHC scoring was done for the markers Ki-67 and PCNA. Correlation of PCNA with Ki67 and tumor grade was performed.

Result: 18 specimens were grade 1 tumors, 36 cases were grade 2 and 16 were grade $3.90 .0 \%$ of tumors were positive for PCNA index and Ki-67 positivity was observed in $85.7 \%$ cases, median and mean values of PCNA and Ki67 were 52.0, 51.64+/-37.475, and 24.50, 26.17士 24.183 respectively. Final score was divided into low and high index using median value as a cut off. Ki-67 LI showed strong positive correlation with histological grade $(\mathrm{p}=0.0001)$. PCNA LI showed positive correlation with histological grade $(\mathrm{p}=0.041)$ but no correlation was observed with Ki-67 LI ( $\mathrm{p}=0.232)$

Conclusion: PCNA marker cannot substitute Ki67 but may be used as an additional marker to assess proliferative activity along with other markers. It may be useful to categorize subgroups with different grades of proliferative activity due to its easy applicability and assessment techniques
\end{abstract}

Keywords: Carcinoma Breast, Histological Tumor Grade, Immunohistochemistry, Ki67, PCNA

\section{Introduction}

Breast cancer ranks first among cancer in urban population of India, and 2nd most common in the rural population. Breast cancer accounts for $25 \%$ to $32 \%$ of all cancers in females. Most people presenting only when symptomatic, and on an average, most 'symptomatic' cancers are stage $2 \mathrm{~B}$ and beyond (significant numbers in stages 3 and 4). India ranks number one in the numbers of healthy life years lost (DALY - Disability Adjusted Life Years) due to breast cancer. Early diagnosis and treatment can reduce breast cancer related morbidity and mortality. ${ }^{[1]}$

Many prognostic factors have been proposed to help identify the patients in whom there is a chance of recurrence after treatment, and in order to select patients with low clinical stage who should receive adjuvant therapy. In addition to histological grading and clinical staging assessment of proliferative activity acts as an adjunct and gives prognostic importance in many tumors including breast cancer. Markers used are mitotic count, AgNor count,
BrDu uptake, Flow cytometry - S phase fraction and Immunohistochemistry (IHC). However, the best method for measurement of proliferative rate is controversial. ${ }^{[2]}$

The simplest method is counting mitotic figures in routine sections, but it has been criticized by others due to subjective variation in identification, different counting protocols and technical pitfalls such as thicker sections and intratumoral difference in distribution. Although determination of labeled thymidine or bromodeoxyuridine correlates well with other prognostic factors the technical complexity limits its usage in routine laboratories. S phase fraction measurement using Flow cytometry has been proven to be a definite prognostic marker in breast carcinomas, however technical difficulty, high cost as well as need of expertise in instrumentation and interpretation are the limiting factors. ${ }^{[3]}$

Ki67 and Proliferating cell nuclear antigen (PCNA) are antigens can be assessed by Immunohistochemistry with the help of monoclonal antibody; they are expressed in 
nucleus of cells during various phases of cell division. Ki67 is expressed in all stages of cell replication and it is an independent prognostic marker, can act as a substitute for mitotic count when assessed along with histological grading. PCNA is $36 \mathrm{KD}$ protein cofactor of DNA polymerase delta, variably present in late $\mathrm{G} 1, \mathrm{~S}$ and mitotic phase of dividing cell. ${ }^{[4]}$

The potential value of IHC marker like PCNA which can be assessed in formalin fixed tissues prompted us to evaluate its positive expression and correlation with other established prognostic markers in seventy untreated breast cancers in mastectomy specimens. The aim was to evaluate the association between PCNA labeling index (PCNA-LI) and Ki67 labeling index (Ki67-LI) and their correlation with histological grade.

\section{Materials and Methods}

This study was conducted in the Department of Pathology in collaboration with Surgery department in JIPMER, Puducherry. Institute ethical committee clearance was obtained; written consent was taken from all the participants. Study is nonfunded. Simple and Modified Radical Mastectomy specimens with the diagnosis of carcinoma breast were included in the study. The specimens treated with neoadjuvant therapy and patients with history of recurrent or relapse was excluded from our study.

Representative samples taken from grossing specimens were fixed in $10 \%$ buffered formalin and those were processed according to routine protocol in Histokinette (Leica TP 1020). Tissues after paraffin embedding, with the help of rotary microtome (Leica RM2125RT) blocks were sectioned into four to six microns thickness sections. Sections were stained by Haematoxyllin and eosin (H\&E) stain. 64 cases were Infiltrating ductal carcinomas NST (no special type), 2 cases were diagnosed as Infiltrating lobular carcinomas, one case of mucinous and comedocarcinoma and two cases were Intracystic papillary carcinoma.

Histological Grading of Tumors: H\&E stained sections were examined for histological grade applying Nottingham's Modification of Bloom Richardson grading system. Grading and IHC scoring was assessed by two authors independently; and were categorized into grade I, II and III. Mitotic count was counted per ten contiguous high power fields in high proliferating areas with good cellularity away from areas of necrosis. In all cases, four sets of ten high power fields were selected and the resultant highest count was taken as the final mitotic index score. ${ }^{[5]}$

Immunohistochemical Study: The monoclonal antibodies used in the present study for IHC are Ki-67 and PCNA (DAKO kits). Streptavidin biotin method including di-amino-benzidine (DAB) as chromogen was applied. Sections taken from paraffin blocks using silane coated slides. Antigen retrieval was done by microwaving; retrieval time of 30 minutes was standardized for Ki-67 and 20 minutes for PCNA. Sections from the lymphnode and skin were used as a positive control for $\mathrm{Ki}-67$ and PCNA respectively.

Analysis of Immunoreactive Scores For Ki-67 and PCNA Markers: ${ }^{[6]}$ The monoclonal antibodies used in the present study for IHC are Ki-67 and PCNA markers. Only unequivocal nuclear staining was considered as positivity for both markers. Minimum thousand cells were counted in consecutive fields to determine final scores. The percentage of positive cells was expressed as the labeling index.

Statistical Analysis Statistical analysis was performed using SPSS software version 21.0. The histological grade and immunohistochemical markers were correlated by nonparametric Spearman's rank correlation coefficient. Chi-square test was applied to assess the association between the parameters. For all statistics, significance was accepted for $p<0.05$.

\section{Results}

Seventy female patients with age ranging from 23 to 76 years, majority of patients fell in the age range of 35 45 years. $34 \%$ of patients were premenopausal and $66 \%$ were postmenopausal. On comparing the results of two authors more than $85 \%$ agreement was obtained. Of the 70 cases 18 were grade 1 tumors, 36 cases were found to be complying with grade 2 and 16 were grade 3 . Photographs of Infiltrating ductal carcinoma (NST) with varying grades showed in Fig.1.Two cases of Papillary carcinoma and Mucinous carcinoma showed grade 1 histology, Infiltrating lobular carcinoma (ILC) and Medullary carcinoma cases showed grade 2 features and Comedocarcinoma was categorized into grade 3. [Fig.2]

Assessment of PCNA Marker Positivity: PCNA showed predominantly diffuse granular and at places mixed positivity of nuclear staining. Though there was significant difference in staining intensity all recognizable positivity was included to give final score. $63(90.0 \%)$ tumours were positive for PCNA index, PCNA index ranged from 0 to $100 \%$ with the median value of 52.0 , mean value with standard deviation is 51.64+/-37.475. Using median value tumors were separated as low and high PCNA index. [Fig.3]

Assessment of Ki-67 Positivity: IHC marker Ki-67 positivity was observed in 60 out of $70(85.7 \%)$ breast 
carcinomas. It was evident as nuclear as well as nucleolar staining. Staining intensity varied among the tumor cells. All cells with recognizable positivity were counted for final score. The assessment of Ki-67 positivity in 70 breast tumours showed their distribution with a median and mean \pm standard deviation of $24.50,29.17 \pm 24.183$ respectively. Final score was divided into low and high index using median value as a cut off. [Fig.4] Then Ki67 LI was correlated with histological grade and PCNA labeling index. ILC and papillary carcinoma types showed decreased IHC markers expression compared with other histological variants. Expression of Ki67 and PCNA markers among different types are depicted in [Fig.5]

Correlation of PCNA with Grade and Ki67: Ki-67 LI showed strong positive correlation with histological grade $(p=0.0001)$. Only one tumour with high Ki-67 LI expression showed grade 1 histology while 14 tumours with high Ki-67 expression were found in grade 3 tumours. Conversely 2 tumours in grade 3 showed a low Ki-67 activity however 17 grade 1 tumours showed low Ki67 LI. There was a significant increase in the mean \pm SD of Ki-67 LI values from low grade to high grade tumors (Table 1).

PCNA LI showed significant positive correlation with histological grade $(p=0.041)$. Six cases with high PCNA LI expression showed grade 1 histology while 12 tumours with high PCNA LI expression were found in grade 3 tumours.
Significant number of high grade tumours was found with high PCNA activity. Conversely four tumours in grade 3 showed a low PCNA activity however 12 grade1 tumours showed low PCNA LI. Significant increase in the mean $\pm \mathrm{SD}$ of PCNA LI values from low grade to high grade tumors were observed. As the tumour grade increased the labeling index value or score of PCNA was observed to be increased, thus giving positive association between the grade and the marker's expression [Table 2]. PCNA index did not show positive correlation with Ki-67 LI ( $p=0.232)$ [scatter plot Fig.5]. It was observed that 21 cases with low PCNA index also showed low Ki67 LI, similarly 20 of high PCNA index cases were found to have high Ki67 LI. Rest of the 29 cases showed either low PCNA LI and high Ki67 LI or vice versa. Hence, though there was very weak correlation between the two, the association is not significant (Table 3). Thus Ki-67 and PCNA marker status of the tumors positively correlated with histological grade. No association was found with PCNA and Ki67 expression.

\section{Discussion}

PCNA is widely used to assess cell proliferation in many malignancies. This marker expression in carcinoma breast patients is extremely variable and the prognostic significance is highly controversial. The present study assessed IHC proliferation markers PCNA and Ki67 expression in seventy carcinoma breast patients and

Table1: Correlation of Ki67 High and Low LI with Histological grade.

\begin{tabular}{|c|c|c|c|c|c|}
\hline \multirow{2}{*}{ Ki - 67 Labeling Index } & \multicolumn{3}{|c|}{ HPE grade } & \multirow{2}{*}{ Chi square test } & \multirow{2}{*}{$P$ value } \\
\hline & Grade1 & Grade 2 & Grade 3 & & \\
\hline Low & 17 & 16 & 2 & \multirow{2}{*}{23.667} & \multirow{2}{*}{0.0001} \\
\hline High & 1 & 20 & 14 & & \\
\hline
\end{tabular}

$p$ value $<0.05$ is considered significant

Table 2: Correlation of PCNA High and Low LI with Histological grade

\begin{tabular}{|l|c|c|c|c|c|}
\hline \multirow{2}{*}{ PCNA Labeling Index } & \multicolumn{3}{|c|}{ HPE grade } & \multirow{2}{*}{ Chi square test } & P value \\
\cline { 2 - 5 } & Grade 1 & Grade 2 & Grade 3 & 6.393 & 0.041 \\
\hline How & 12 & 20 & 4 & 12 & \multirow{2}{*}{ High } \\
\hline
\end{tabular}

$p$ value $<0.05$ is considered significant

Table 3: Correlation of PCNA High and Low LI with Ki67 High and Low LI.

\begin{tabular}{|c|c|c|c|c|}
\hline & \multicolumn{2}{|c|}{ PCNA labelling index } & \multirow{2}{*}{ Chi square test } & \multirow{2}{*}{$P$ value } \\
\hline & Grade 1 & High & & \\
\hline Ki67 Labeling Index & Low & 14 & \multirow{2}{*}{2.059} & \multirow{2}{*}{0.232} \\
\hline Low & 21 & 20 & & \\
\hline High & 15 & & & \\
\hline
\end{tabular}

$p$ value $<0.05$ is considered significant 


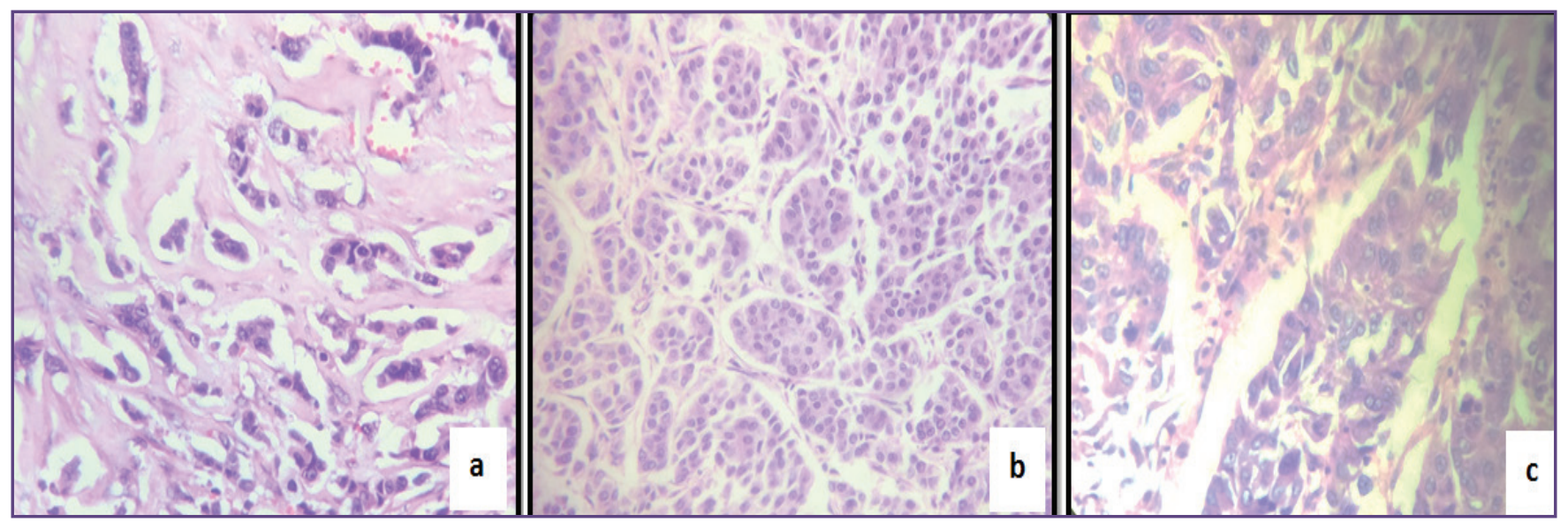

Fig. 1: Showing different grades of Infiltrating ductal carcinoma NST (H\&E) a)Grade 1-score 3, (x 400), b)Grade 2- score 6, (x 100), c) Grade 3- score 8, (x 400).

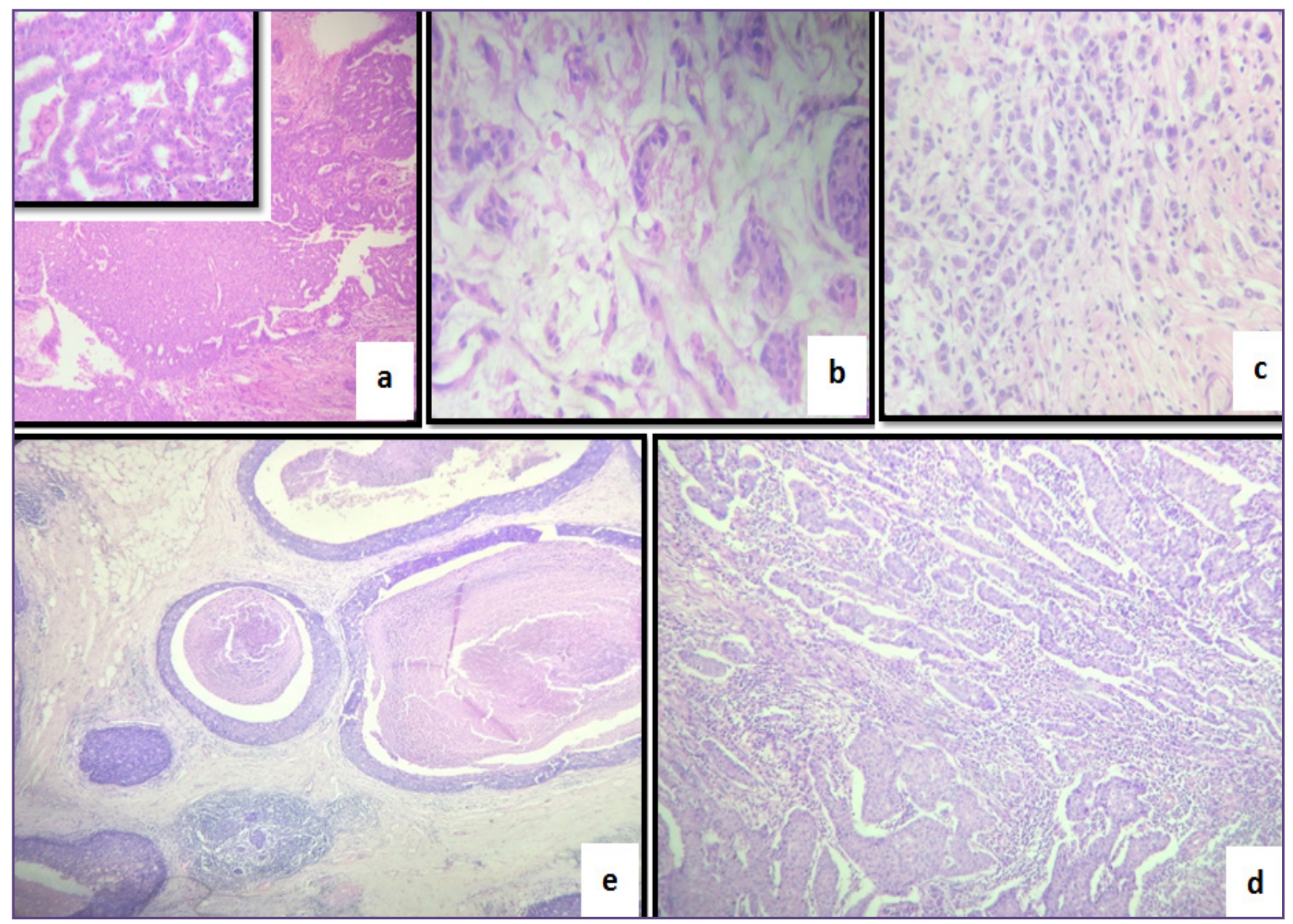

Fig. 2: demonstrates histopathology pictures of infiltrating ductal carcinoma, special types in varying grades (H\&E) (a) Intracystic papillary carcinoma Grade 1, score 5, inset- anastamosing papillary processes (x 40) b) Mucinous carcinoma, Grade 1, score 5, (x 100) c) Infiltrating lobular carcinoma, Grade 2, score 6, (x 100) d) Medullary carcinoma, Grade 2, score 6, e) Comedocarcinoma, Grade 3, score 8(x 40). 


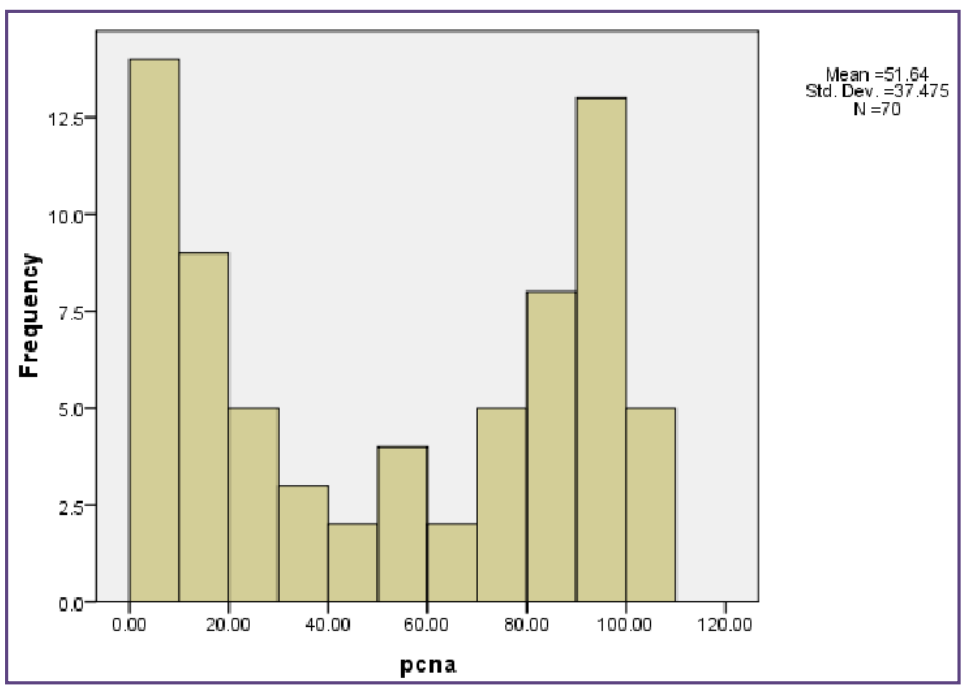

Fig. 3: Frequency distribution of PCNA among the study group [N=70], Median value-52.0

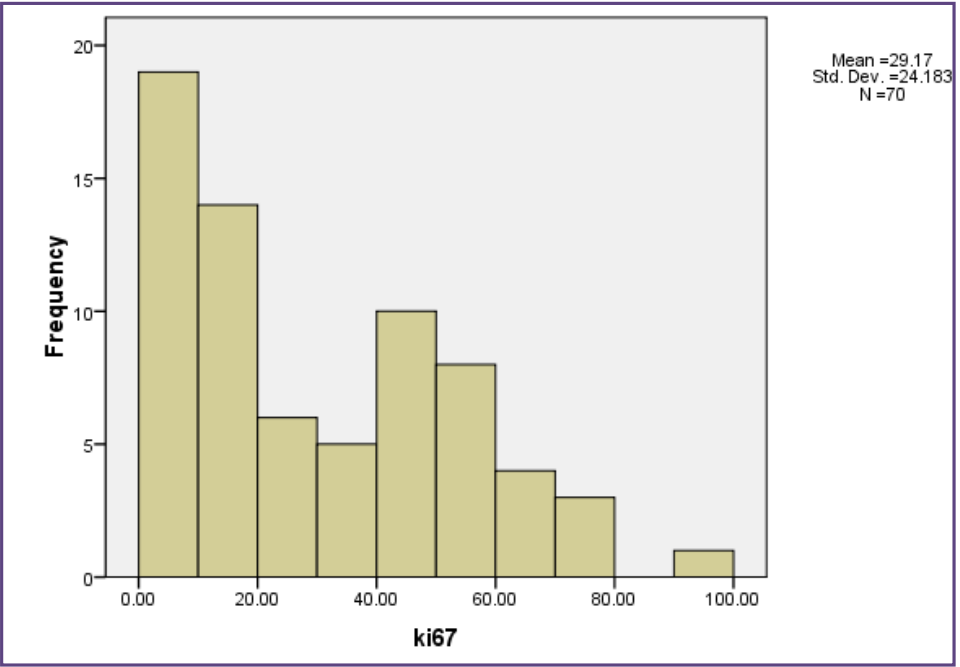

Fig. 4: Frequency distribution of Ki67 among the study group [N=70], Median value 24.5.

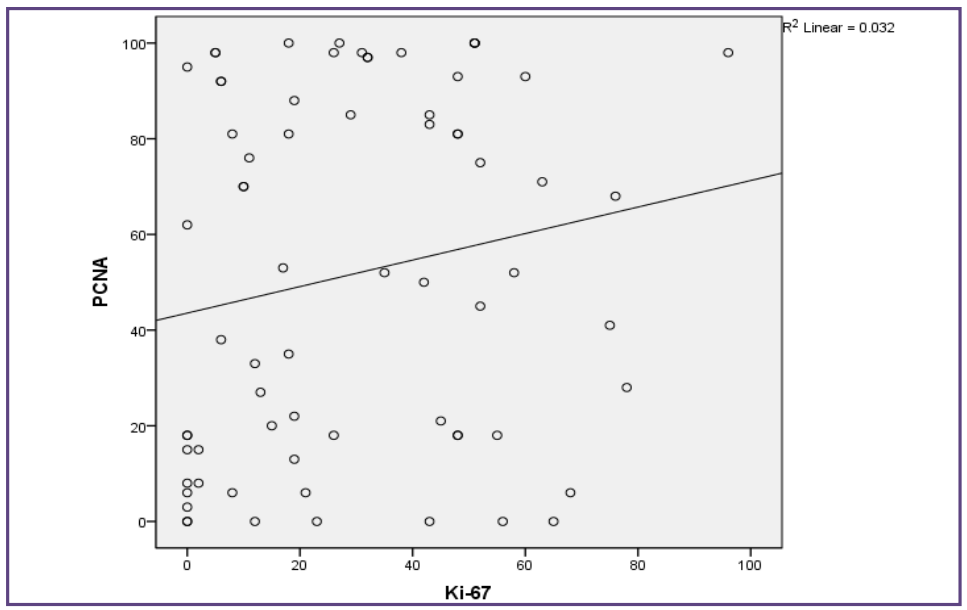

Fig. 5: Scatter plot of PCNA LI against Ki67 LI, No significant correlation observed. 
evaluated the association between these markers as well as with the established independent prognostic marker histological grade.

Schonborn et $\mathrm{al}^{[7]}$ studied 471 breast cancer cases and showed that in univariate analysis with a median follow up of 5 years, survival rates depended on PCNA expression in node negative patients other than histological grade, and they concluded that PCNA expression was a valuable prognostic marker. Conversely Masakuni Noguchi ${ }^{[8]}$ studied 91 patients with operable invasive breast cancer patients and showed that PCNA expression did not correlate with clinicopathologic and biologic prognostic factors. The results of previous larger studies were highly contradictory.

Our study showed significant positive correlation between PCNA and histological grade and highly significant association between Ki67 LI and grade. Similar results were identified by other authors also ( Pelosi G and Brown DC). ${ }^{[9,10]}$ The Ki67 LI mean and median values agree with already reported values ranging from 7.2 to $32.4 \%$, the highest median value of $32.4 \%$ was observed by study done by Caly M. ${ }^{[11]}$ When tumor grade is high the resultant proliferative activity is also higher which is reflected by high LI of PCNA and Ki67. Our results indicate that high grade tumors follow uncontrolled proliferative activity as a mechanism of tumor progression.

Additional advantage of this marker is easy applicability in routine formalin fixed tissues, frozen sectioning is not necessary. However, due to heterogeneous staining quality, to exclude subjective variation in the assessment of LI, formulation of standardized scoring system or automated image analysis is suggested.

The lack of association between PCNA and Ki67 LI on breast carcinoma is surprising. Our results correlate with the study done by Leonardi et al and Hall et $\mathrm{a}^{[12,13]}$, who showed similar insignificant association between these markers. Similar results were also obtained by Thomas et al and Surowik et al ${ }^{[14,15]}$ who studied correlation of these two markers in good number of primary breast carcinomas, our results agree with their conclusions. But, these results contradict the data obtained by Pelosi G et al, Dawson et al and Battersby et a $\mathrm{a}^{[9,16,17]}$ who showed positive correlation between these two markers. However they applied different antibody lineage as well as used frozen sections in their study. These contrasting results may be attributed to high sensitivity of PCNA marker to fixation time and antigen retrieval techniques, which varies widely among laboratories.

PCNA expression is higher than Ki67 expression in our study as other previous studies (Pelosi G) $\cdot{ }^{[9]}$ In contrast to
Ki67 which is expressed only in cells actively involved in cell cycle, PCNA is frequently over expressed in noncyclical cells also in variable manner. The discrepancy of PCNA LI may be due to its role in DNA repair in addition to cell proliferation. So, the final LI not only indicates proliferation, it includes the damaging cells undergoing cell repair, in turn the resultant values are higher than the values expected. Higher median value of PCNA in comparison with the median value of Ki67 is attributed to prolonged half life and persistence of the antigen in cells which have already completed cell cycle. ${ }^{[18,19]}$

\section{Conclusion}

Our data showed significant association of PCNA LI to histological grade; however the association is not as strong as Ki67 LI towards tumor grade. Though we observed very week correlation between PCNA and Ki67 LI, there was lack of significant association between these two proliferative markers. PCNA marker is influenced by other undefined factors to larger extent that it affects the usefulness and prognostic significance of this marker in assessing proliferative activity in breast cancer patients.

Though PCNA LI is not comparable with the Ki67 LI, this marker exhibits significant correlation with the grade which is a well proven independent prognostic marker. Hence it cannot substitute Ki67 but may be used as an additional marker to assess proliferative activity along with other markers. It may be useful to categorize subgroups with different grades of proliferative activity due to its easy applicability and assessment techniques. To know the definitive role of PCNA as a useful proliferative marker in breast carcinoma patients, large clinical follow up of such patients in large sample size should be studied considering disease free period and survival data.

\section{Acknowledgements}

We would like to thank the technical staff Mrs. Girija, IHC lab, and Dr. Jayanthi, Professor and Head of the Department of Pathology, JIPMER for the general help.

\section{Reference:}

1. Lyon, France: International Agency for Research on cancer. Bray F, Ren JS, Masuyer E, Ferlay J. Global estimates of cancer prevalence for 27 sites in the adult population in 2008. Int J cancer, 2013;132(5):1133-45.

2. Sullivan RP, Mortimer G, Muircheartaigh IO. Cell proliferation in breast tumours: analysis of histological parameters Ki67 and PCNA expression. Ir J Med Sci. 1993; 162(9):343-7.

3. Albert A. Keshgegian, Avital cnaan. Proliferation Markers in Breast Carcinoma Mitotic Figure Count, S-Phase Fraction, Proliferating Cell Nuclear Antigen, Ki-67 and MIB-1. Am J Clin Pathol 1995;104:42-49. 
4. Gerdes J, Lemke H, Baisch H, Wacker HH, Schwab U, Stein $\mathrm{H}$. Cell cycle analysis of a cell proliferation-associated human nuclear antigen defined by the monoclonal antibody Ki-67. J Immunol. 1984; 133(4):1710-5.

5. Simpson, JF, Gray R, Dressler LG, Cobau CD, Falkson CI, Gilchrist KW. et al. Prognostic value of histologic grade and proliferative activity in axillary node-positive breast cancer: results from the Eastern Cooperative Oncology Group Companion Study, EST 4189. J Clin Oncol 2000; 18: 2059-69

6. Van Diest, Paul J, Brugal, Gerard, Baak, Jan PA. Proliferation markers in tumours: interpretation and clinical value J Clin Pathol 1998; 51(10): 716-724.

7. Schönborn I, Minguillon.C, Möhner.M, Ebeling . PCNA as a potential prognostic marker in breast cancer, breast Volume 3, Issue 2, June 1994, Pages 97-102

8. Noguchi, Thomas MM, Kitagawa H, Kinoshita K, et al. The prognostic significance of proliferating cell nuclear antigen in breast cancer: correlation with DNA ploidy, c-erbB-2 expression, histopathology, lymph node metastases and patient survival. Int. J. Oncol. 2: 985-989. 1993

9. Pelosi G, Bresaola E, Rodella S, Manfrin E, Piubello Q, Schiavon I, Iannucci A. Expression of proliferating cell nuclear antigen, Ki-67 antigen, estrogen receptor protein, and tumor suppressor p53 gene in cytologic samples of breast cancer: an immunochemical study with clinical, pathobiological, and histologic correlations. Diagn Cytopathol. 1994;11(2):131-40

10. Brown DC, Gatter KC. Monoclonal antibody Ki67: its use in histopathology. Histopathology 1990;17:489-503.

11. Caly M, Genin P, Ghuzlan AA. Analysis of correlation between mitotic index, MIB1 score and S-phase fraction as proliferation markers in invasive breast carcinoma.
Methodological aspects and prognostic value in a series of 257 cases. Anticancer Res 2004; 24: 3283-88.

12. Leonardi E, Girlando S, Serio G. PCNA and Ki-67 expression in breast carcinoma; Correlation with clinical biological variables. J Clin Patrhol 1992; 45: 416-419.

13. Hall PA, Levison DA, Woods AL, et al. Proliferating cell nuclear antigen (PCNA) immunolocalization in paraffin sections: an index of cell proliferation with evidence of deregulated expression in some neoplasms. J Pathol 1990;162:285-94.

14. Thomas M, Noguchi M, Kitagawa H, Kinoshita $K$, Miyazaki I Poor prognostic value of proliferating cell nuclear antigen labeling index in breast carcinoma. J Clin Pathol 1993; 46: 525-528

15. Surowiak P, Pudelko M, Maciejczyk A. The relationship of the expression of proliferetron--related antigens Ki67 and PCNA in the cells of ductal breast cancer with the differentiation grade. Ginekol Pol 2005; 76: 9-14.

16. Dawson AE, Norton JA, Weinberg DS. Comparative assessment of proliferation and DNA content in breast carcinoma by image analysis and flow cytometry. Am J Pathol 1990;136:1 115-24.

17. Battersby S, Anderson TJ. Correlation of proliferative activity in breast tissue using PCNA/cyclin. Hum Pathol 1990;21:781.

18. Mercer EW, Shield MT, Amin M, et al. Negative growth regulation in a glioblastoma tumor cell line that conditionally expresses human wild-type p53. Proc Natl Acad Sci USA 1990;87:6166-70.

19. Haneda H, Katabami M, Miyamoto H, et al. The relationship of the proliferating cell nuclear antigen protein to CDDP resistance of a murine leukemia cell line P388/CDDP. Oncology 1991;48:234-8.

*Corresponding author:

Kalaivani Amitkumar, S1, Thanya regency, 11/61 Ganesh Nagar Main Road, Selaiyur, East Tambaram, Chennai-600 073, Tamil Nadu, India.

Phone: +91 9843575500

Email: drkalaivani1980@gmail.com

Date of Submission : 04.12.2016

Date of Acceptance : 20.12.2016

Financial or other Competing Interests: None.

Date of Publication : 23.02.2017 